\title{
Integración de las TIC en la enseñanza aprendizaje en línea de la asignatura matemática del ITB
}

\section{Integration of ICT in teaching online learning of the ITB Math Subject}

\author{
Luisa Toyo Sánchez ${ }^{1}$ \\ Imtoyo@itb.edu.ec \\ ORCID: https://orcid.org/0000-0003-4822-7055
}

Recibido:07/12/2020, Aceptado: 26/03/2021

\begin{abstract}
RESUMEN
La integración de las TIC en la enseñanza aprendizaje en línea del Instituto Superior Tecnológico Bolivariano de Tecnología, tiene el propósito institucional del uso de tecnologías interactivas multimedia y entornos virtuales. Sin embargo, carecen de recursos educativos abiertos para el dictado de la asignatura matemática, siendo una asignatura práctica, de alto nivel de abstracción, que requiere el acompañamiento a los docentes en este proceso de transformación educativa para los estudiantes. El objetivo del presente trabajo fue determinar la integración de las TIC en el proceso de la enseñanza aprendizaje en línea de la asignatura matemática. Para ello se planteó una metodología mixta porque el levantamiento de la información basado en la revisión documental y en la observación directa del Entorno Virtual de Aprendizaje para generar información, así como en la evidencia de datos numéricos provista por el cuestionario de encuesta dirigido a docentes y estudiantes, todo lo cual permite una interpretación integral del fenómeno bajo estudio. Como resultado se obtuvo que la gran mayoría utiliza las TIC en sus clases, lo cual está vinculado a la modalidad de estudios de su carrera. Sin embargo, no hay un uso óptimo de la plataforma y de las herramientas tecnológicas. Se concluye que utilizar recursos educativos abiertos en la red que permiten mejorar la formación académica de los estudiantes en la asignatura de matemáticas, permite facilitar la interacción de una manera más didáctica y personalizada para cada tipo de estudiante, logrando que siempre se genere la enseñanza basada en TIC.
\end{abstract}

Palabras clave: Integración, TIC, enseñanza, aprendizaje

\section{ABSTRACT}

The integration of ICT in online teaching and learning at the Bolivarian Higher Technological Institute of Technology has the institutional purpose of using interactive multimedia technologies and virtual environments. However, they lack open educational resources for the dictation of the mathematical subject, being a practical subject, with a high level of abstraction, which requires the

\footnotetext{
${ }^{1}$ Magister en Educación, docente del Instituto Superior Tecnológico Superior Bolivariano, Ecuador.
} 


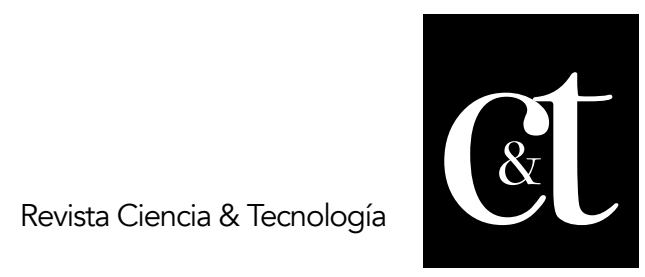

No. 30, 30 de abril de 2021

ISSN impreso: 1390 - 6321

ISSN online: 2661 - 6734

accompaniment of teachers in this process of educational transformation for students. The objective of this work was to determine the integration of ICT in the online teaching-learning process of the mathematical subject. For this, a mixed methodology was proposed because the collection of information based on the documentary review and direct observation of the Virtual Learning Environment to generate information, as well as on the evidence of numerical data provided by the survey questionnaire directed to teachers and students, all of which allows a comprehensive interpretation of the phenomenon under study. As a result, the vast majority had to use ICT in their classes, which is linked to the type of study in their career. However, there is no optimal use of the platform and technological tools. It is concluded that using open educational resources on the network that allow improving the academic training of students in the subject of mathematics, allows to facilitate interaction in a more didactic and personalized way for each type of student, achieving that based teaching is always generated in ICT.

Keywords: Integration, ICT, teaching, learning

\section{Introducción}

Actualmente, la necesidad del hombre por presentar y percibir su entorno, ha generado en la sociedad la búsqueda del conocimiento, aquel componente necesario que lo ayude a comprender su realidad individual y social. Para Chaparro (2001) la posición del hombre dentro de la sociedad se vislumbra como aquella, donde el individuo será capaz de adquirir y generar conocimiento que le permita adaptarse a una realidad dinámica y en constante cambio. Es por esto, que proporcionar un entorno educativo en el que puedan desarrollarse, de manera efectiva, nuestros estudiantes están en manos de los educadores.

Realmente, se necesita un entorno con una innovación pedagógica en profundidad y con un cambio que beneficie las tecnologías de la información y la comunicación (TIC). Así como plantea el Plan Nacional del Desarrollo que se deben implementar modalidades alternativas de educación para la construcción de una sociedad educadora en los niveles que mayor atención requieren: el bachillerato y la educación superior, para tener un abanico de opciones de estudios y los estudiantes puedan alcanzar su nivel profesional, siendo la educación en línea una de ellas.

Para ello, se indica que dicha modalidad es parte de la cuarta y quinta generación de la educación a distancia, debido al uso de las TIC y principalmente del internet para expandir la era del conocimiento e implementar modelos educativos acorde con las exigencias de la sociedad actual. Por su parte, García (2007) indica que las posibilidades educativas del ciberespacio son las que determinan el paso de una educación a distancia a una educación virtual, en donde el profesional debe ser capaz de adaptarse a los avances del mundo actual y por lo tanto un requisito esencial en estos días es ingresar a la era de la educación en línea.

Toyo. Integración de las TIC en la enseñanza aprendizaje en línea de la asignatura matemática del ITB 


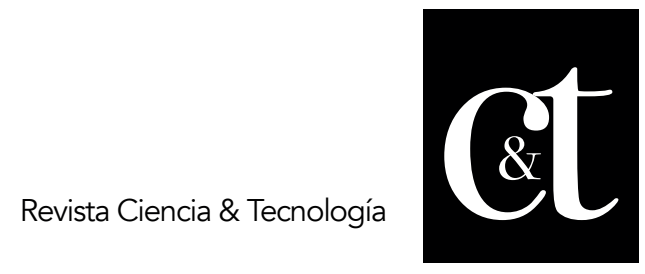

No. 30, 30 de abril de 2021

ISSN impreso: 1390 - 6321

ISSN online: 2661 - 6734

Ecuador, se suma a este cambio y es por eso que en el 2018 las universidades comienzan a insertarse en esta modalidad regulados por la Secretaria de Educación Superior, Ciencia, Tecnología e Innovación (Senescyt) brindando un programa de educación superior virtual para los bachilleres para diversificar la oferta educativa contando con universidades que tengan esta modalidad.

Si bien es cierto, estos nuevos contextos vienen caracterizados por la presencia de ordenadores, materiales didácticos multimedia, Internet, comunicaciones asíncronas y síncronas o plataformas de e-learning por lo que son variados los factores didácticos, económicos y sociológicos que caracterizan las nuevas relaciones y problemas educativos. Pero la nueva tecnología se ha ido implantando en las Instituciones sin alterar en muchos casos, el orden tradicional de su práctica y de los modelos de transmisión del conocimiento, compatible con muchos estilos de enseñanza y respondiendo a intereses económicos más que a una intencionalidad renovadora, usando las tecnologías del mañana para administrar el currículum del pasado.

El Instituto Superior Tecnológico Bolivariano de Tecnología, siendo concebido para la docencia, investigación y extensión, ha forjado sus esfuerzos en la formación no solamente de sus estudiantes, sino también la transmisión de sus logros a la sociedad, pero requiere la incorporación de las tecnologías para mejorar la enseñanza hacia los estudiantes.

Esto supone que debe tener la capacidad para producir conocimientos innovadores, donde la generación del conocimiento individual incremente el capital intelectual de los profesores y el capital intelectual de la institución. Las Tecnologías de la Información y las Comunicaciones (TIC) han venido a proporcionar esas herramientas de indudable valor para la generalización de bases de conocimiento $y$, en este sentido, constituyen un nuevo ámbito de investigación y desarrollo a nivel superior.

Cabe recalcar, que el modelo educativo que utiliza el instituto es basado en competencias, siendo la combinación de destrezas, conocimientos, aptitudes y actitudes, y a la inclusión de la habilidad para aprender además del saber cómo, con la finalidad de que los estudiantes logren desarrollar capacidades para resolver problemas, orientando a los estudiantes según las necesidades que puedan tener en la enseñanza aprendizaje en línea.

El Instituto Superior Tecnológico Bolivariano de Tecnología, actualmente cuenta con la carrera de Tecnología Superior en Administración bajo la modalidad en línea, con el propósito institucional del uso de tecnologías interactivas multimedia y entornos virtuales para el proceso de enseñanza aprendizaje, no obstante carecen de recursos educativos abiertos para impartir la asignatura de matemática, siendo esta una asignatura práctica, de alto nivel de abstracción, que requiere tener mejor interacción entre docente alumno.

La implementación de estos recursos permite que los estudiantes usen las tecnologías para generar el proceso de enseñanza aprendizaje en línea de la asignatura matemática, por medio de estrategias didácticas adecuadas y de útiles herramientas tecnológicas orientadas por los docentes a través de recursos educativos abiertos por medio de la plataforma Moodle. 


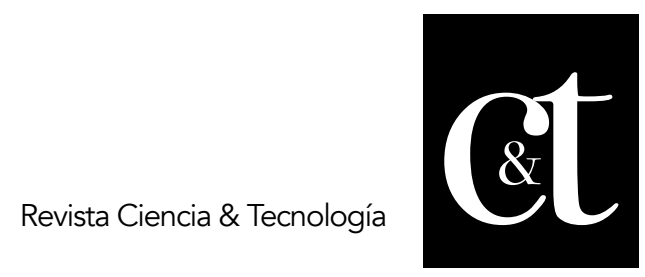

No. 30, 30 de abril de 2021

ISSN impreso: 1390 - 6321

ISSN online: 2661 - 6734

Es así, que es de gran aporte esta investigación ya que resalta el proceso de enseñanza-aprendizaje a través de las tecnologías de la información y comunicación como soporte en la interacción con actividades didácticas que integran lo visual, novedoso e interactivo; impulsa el uso de aplicaciones, plataformas y redes sociales; promueve nuevas formas de enseñanza; facilita la búsqueda de información y comunicación, el desarrollo de actividades prácticas del quehacer docente como las videoconferencias.

\section{Metodología}

Para el presente estudio, se utilizó una investigación mixta en donde según Hernández, Fernández y Baptista (2014), la meta de la investigación mixta no es remplazar a la investigación cuantitativa ni a la investigación cualitativa, sino utilizar las fortalezas de ambos tipos de indagación combinándolas y tratando de minimizar sus debilidades potenciales. Así mismo, el objeto de estudio es aplicada ya que busca la utilización de los conocimientos adquiridos, a la vez que se adquieren otros.

La investigación es de campo, siendo realizada en el sitio donde se produce el fenómeno en Instituto Superior Tecnológico Bolivariano de Tecnología, para tomar contacto directo con la realidad, de cómo se lleva a cabo los procesos de enseñanza aprendizaje virtual. De igual manera bibliográfico, sustentando teórica y conceptualmente la investigación mediante la revisión de literatura científica (libros, revistas, manuales) y el EVA que permitiendo analizar y contractar los resultados obtenidos en el presente estudio

Se utilizó la técnica documental con el instrumento ficha de registro en donde el investigador tiene acceso a dicha información que está en el Entorno Virtual de Aprendizaje, de igual manera la técnica de la encuesta, con el instrumento cuestionario, que fueron analizadas por el experto tanto en investigación como del área y temas investigados, dirigido a docentes y estudiantes, que facilitaron la información. Siendo enviada por medio de un link (google forms) a cada miembro de dicha población, en donde luego fue analizada e interpretada.

La población está constituida por 30 estudiantes de la carrera de Tecnología Superior en Administración, cursantes de la asignatura matemática de la modalidad en línea, con un rango de edad de 17 a 45 años, siendo 20 mujeres y 10 hombres. Adicionalmente, 05 docentes ( 04 hombres y 01 mujer) en el área de matemática. Considerando que la población objeto de análisis es pequeña se tomó toda la población, para una muestra no probabilística decisional, con un total de 30 estudiantes y 05 docentes. Todos los cuales dieron su consentimiento informado para participar en el estudio.

Toyo. Integración de las TIC en la enseñanza aprendizaje en línea de la asignatura matemática del ITB 


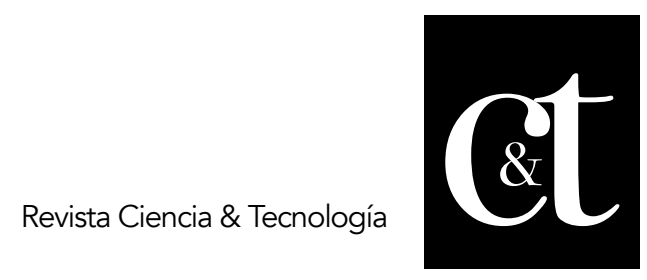

No. 30, 30 de abril de 2021

ISSN impreso: 1390 - 6321

ISSN online: 2661 - 6734

Tabla1. Población de estudiantes

\begin{tabular}{|ccc|}
\hline $\begin{array}{c}\text { Tabla1. Población de estudiantes } \\
\text { Unidades de observación }\end{array}$ & No. \\
\hline Estudiante Género Mujer & 20 & 66 \\
& & 6 \\
& & 6 \\
\hline Estudiante Género Hombre & 10 & 33 \\
& & 3 \\
\hline Total & 30 & 10 \\
\hline
\end{tabular}

Fuente: Elaboración propia

Tabla 2. Población de Docentes

\begin{tabular}{|lll|}
\hline Unidades de observación & No. & $\%$ \\
\hline Docente Género Mujer & 1 & 20 \\
\hline Docente Género Hombre & 4 & 80 \\
\hline Total & $\mathbf{5}$ & $\mathbf{1 0 0}$ \\
\hline
\end{tabular}

Fuente: Elaboración propia

\section{Resultados y discusión}

Tecnologías de la Información y Comunicación (TIC)

Se denominan TIC, según Rosario (2005), al conjunto de tecnologías que permiten la adquisición, producción, almacenamiento, tratamiento, comunicación, registro y presentación de informaciones, en forma de voz, imágenes y datos contenidos en señales de naturaleza acústica, óptica o electromagnética. Ellas incluyen la electrónica como tecnología base que soporta el avance de las telecomunicaciones, la informática y el audiovisual. De este modo, las Tecnologías de la Información y Comunicación han permitido llevar la globalidad al mundo de la comunicación, facilitando la interconexión entre las personas e instituciones a nivel mundial, y eliminando barreras espaciales y temporales.

Estrategias de enseñanzas basadas en TIC

El uso de estrategias basadas en TIC, promueve aprendizajes significativos en los estudiantes, debido a la alta estimulación que genera en los mismos el uso de recursos tecnológicos de su entorno, teniendo en cuenta su condición de nativos digitales. Es necesario considerar que la acelerada inserción de las nuevas tecnologías en el ámbito educativo genera nuevos retos para las instituciones educativas, entre ellos, describe (Área, 2009):

- Integrar las nuevas tecnologías en el sistema y cultura escolar.

- Reestructurar los fines y métodos de enseñanza. Nuevos roles para docentes y estudiantes.

- Extender la formación a través de las redes.

- Revisar y replantear la formación ocupacional a la luz de las nuevas exigencias socio- laborales impulsadas por las nuevas tecnologías. 


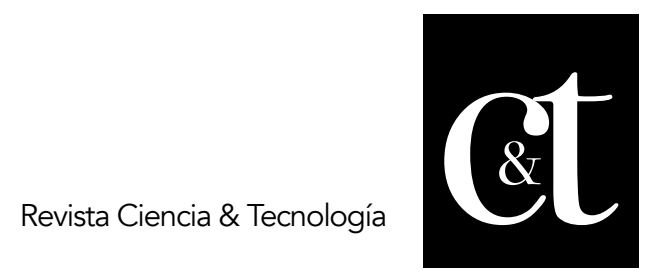

No. 30, 30 de abril de 2021

ISSN impreso: 1390 - 6321

ISSN online: 2661 - 6734

Las instituciones educativas deben obtener el reto de integrar las nuevas tecnologías con calidad en su contexto, para no quedar rezagados ante los avances de la sociedad globalizada en la que se desenvuelven los estudiantes de hoy dando seguridad en el proceso de enseñanza aprendizaje.

\section{Recursos educativos didácticos}

El significado de recursos educativos didácticos se le ha llamado de diversos modos, como: apoyos didácticos, recursos didácticos, medios educativos. Según Morales (2012), se entiende por recurso didáctico al conjunto de medios materiales que intervienen y facilitan el proceso de enseñanza-aprendizaje. Estos materiales pueden ser tanto físicos como virtuales, asumen como condición, despertar el interés de los estudiantes, adecuarse a las características físicas y psíquicas de los mismos, además que facilitan la actividad docente al servir de guía; asimismo, tienen la gran virtud de adecuarse a cualquier tipo de contenido.

La importancia del material didáctico radica en la influencia que los estímulos a los órganos sensoriales ejercen en quien aprende, es decir, lo pone en contacto con el objeto de aprendizaje, ya sea de manera directa o dándole la sensación de indirecta. Las funciones que tienen los recursos didácticos toman en cuenta el grupo al que va dirigido, con la finalidad que ese recurso realmente sea de utilidad. En la figura 1 se detallan tales funciones de los recursos didácticos.

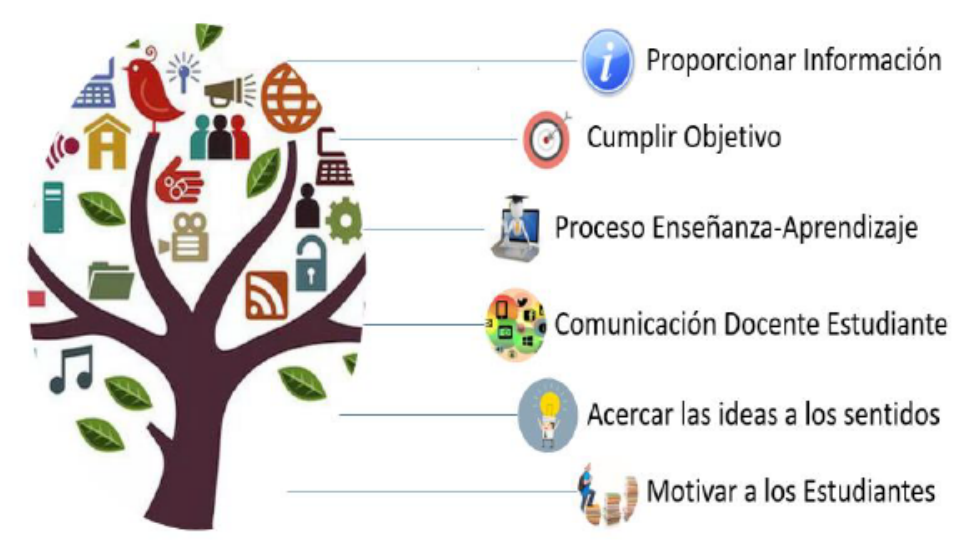

Figura 1. Recursos Didácticos

Fuente: Elaboración propia con base en Morales (2012)

Las figuras 2 y 3 muestran los tipos de recursos didácticos de textos impresos y material audiovisual, respectivamente, mientras que la figura 4 detalla los tableros didácticos.

Toyo. Integración de las TIC en la enseñanza aprendizaje en línea de la asignatura matemática del ITB 


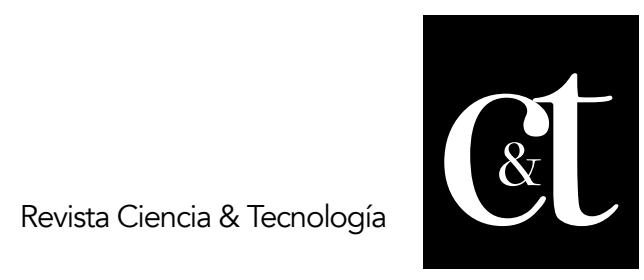

No. 30, 30 de abril de 2021

ISSN impreso: 1390 - 6321

ISSN online: 2661 - 6734

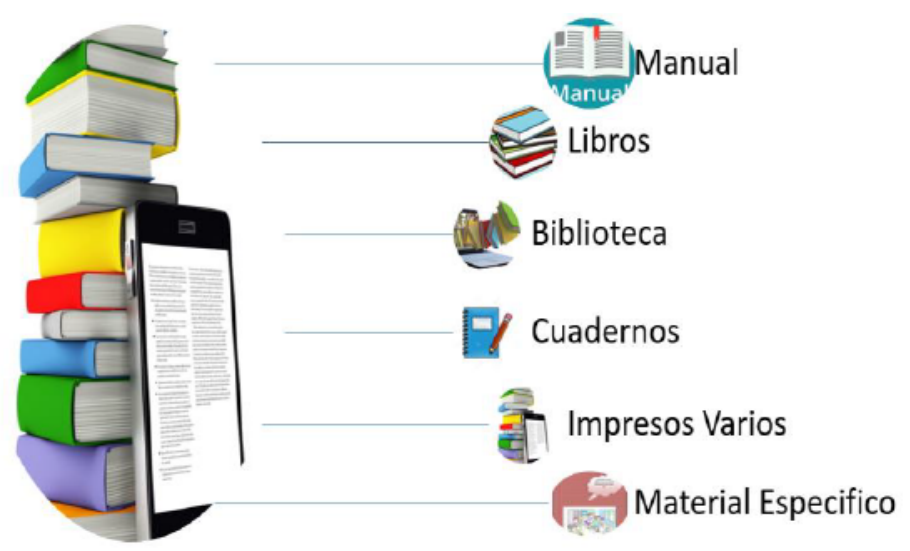

Figura 2. Textos Impresos

Fuente: Elaboración propia con base en Moya (2010)

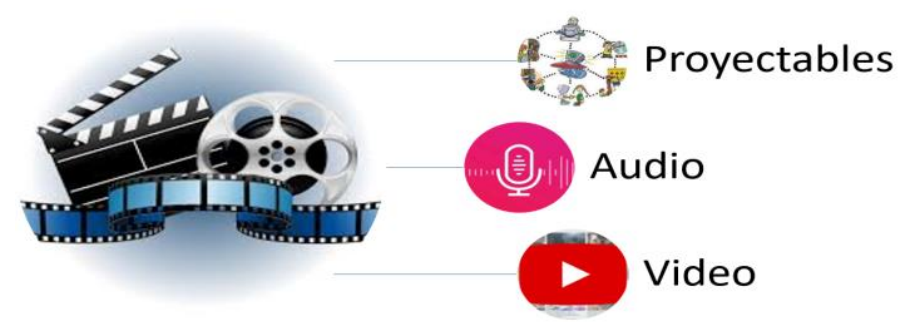

Figura 3. Material audiovisual

Fuente: Elaboración propia con base en Moya (2010)

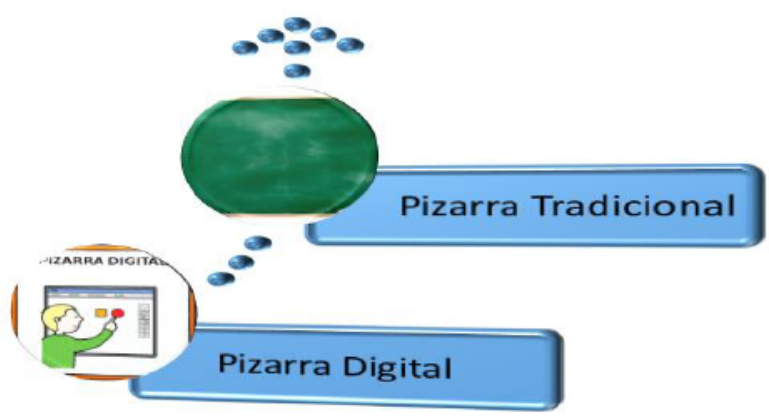

Figura 4. Tableros didácticos

Fuente: Elaboración propia con base en Moya (2010)

\section{Herramientas Tecnológicas}

Para Cabero y Llorente (2005), las plataformas virtuales son ordenadores que se utilizan para la creación, gestión y distribución de actividades formativas a través de la Web: son aplicaciones que facilitan la creación de entornos de enseñanza aprendizaje, integrando materiales didácticos y herramientas de comunicación, colaboración y gestión educativa. 


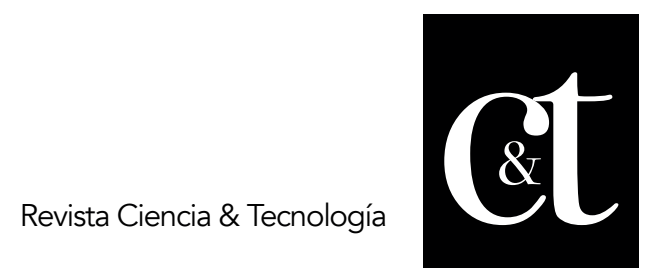

No. 30, 30 de abril de 2021

ISSN impreso: 1390 - 6321

ISSN online: 2661 - 6734

En donde, por medio de la e-learning brindan ambientes de aprendizaje ya diseñados e integrados. A ellos acceden los alumnos a través de una clave personal. Por ello, se trata de un espacio privado, dotado de herramientas necesarias para aprender.

En cuanto a Hot Potatoes, es un sistema para organizar ejercicios educativos que pueden realizar posteriormente a través de la web. Los ejercicios que crea son del tipo respuesta corta, selección múltiple, rellenar los huecos, crucigramas, emparejamiento y otros. Su licencia no es libre, pero permite su uso a instituciones educativas públicas sin ánimo de lucro siempre que los resultados sean accesibles vía web (Lázaro, Ruiz y González , 2009),

Adell (2004) define la WebQuest como un tipo de actividad didáctica que consiste en una investigación guiada, con recursos principalmente procedentes de Internet, que promueve la utilización de habilidades cognitivas superiores, el trabajo cooperativo y la autonomía de los alumnos e incluye una evaluación auténtica.

Autores como Chenoweth, Abril y Meza (2017), plantean que H5P es un marco de trabajo colaborativo de contenidos libre y de fuente abierta basado en JavaScript. Permite crear imágenes, presentaciones, líneas de tiempo, escenarios y videos interactivos, tour virtual, test de personalidad, cuestionarios, entre otros. Tiene tres propuestas claras: crear contenido HTML5 enriquecido para plataformas de publicación existentes, compartir el contenido entre sitios que soporten H5P, reutilizar y modificar el contenido desde un navegador de internet las veces que sean necesarios.

Por su parte, Games es un editor y revelador de software para juegos de computadoras ocasionales y tradicionales que pueden ser adaptadas al ámbito de la educación. La cual proporciona el registro y la ayuda técnica necesaria para usarlo de manera adecuada (Bertran y Chamarro, 2016).

Finalmente, la herramienta Blog es un sitio web de actualización constante, donde se publican cronológicamente textos o artículos de uno o varios autores. Los usuarios pueden leer los aportes o entradas y dejar su comentario. Al organizar un blog para un curso, los estudiantes pueden anunciar sus artículos, resultado investigaciones asignadas, también se pueden utilizar como portafolio de actividades. Entre las herramientas para crear blogs están: WordPress y Blogger (Fernández, 2018).

Las herramientas para regenerar la experiencia de aprendizaje son muchas, a su vez algunas gratuitas, flexibles y fáciles de utilizar. Sin embargo, las herramientas no son el fin, son solo un medio para facilitar el aprendizaje y generar habilidades informáticas necesarias para los estudiantes. Éstas no reemplazan al tutor virtual, al contrario, le permiten transformar su rol, por uno más participativo, dinámico y facilitador.

Toyo. Integración de las TIC en la enseñanza aprendizaje en línea de la asignatura matemática del ITB 


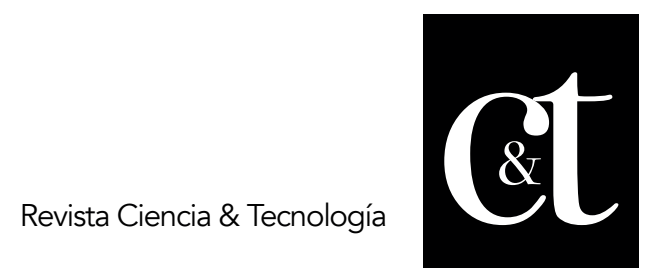

No. 30, 30 de abril de 2021

ISSN impreso: 1390 - 6321

ISSN online: 2661 - 6734

\title{
Modalidades de Estudio
}

\begin{abstract}
Virtual
Lara (2002), define la educación virtual como la modalidad educativa que eleva la calidad de la enseñanza-aprendizaje, y esto debido a que respeta su flexibilidad o disponibilidad, es decir, se puede canalizar para tiempos y espacios variables. Según el autor, esta modalidad logra su mayor reconocimiento con la tecnología a través de los métodos asincrónico, sincrónico y autoformación. Cabe mencionar que cada Institución se rige por un total de 30 a 40 horas de estudio semanal, de acuerdo al nivel de educación; conformadas por un $70 \%$ a $80 \%$ de clases virtuales impartidas por los docentes (sincrónico) y $30 \%$ a $20 \%$ de actividades de comprensión off line (asincrónico), apoyadas por los docentes.
\end{abstract}

\section{Semi-Presencial}

Es una modalidad educativa que demanda un mínimo de horas de clases presenciales y el resto del tiempo se define como estudio independiente, periodo en el cual el estudiante cumplirá con las asignaciones encomendadas por el docente accediendo a la plataforma virtual de la Institución, realizando investigación a través de fuentes tradicionales o electrónicas. Para aprobar los cursos bajo esta modalidad, es requerimiento obligatorio cumplir con un mínimo de asistencia a los encuentros presenciales físicos en el aula o laboratorio (Silva y Calichs, 2013).

\section{Presencial}

Es una modalidad donde la figura del profesor inmediato es la base de este tipo de educación. (Andersen, 1979). "El profesor inmediato es conceptualizado como los comportamientos no verbales que reducen la distancia física y psicológica entre los maestros y los estudiantes" (p. 544). Se mantiene una relación estrecha con docentes y compañeros de clases, lo cual es un elemento motivacional para el para el proceso de enseñanza aprendizaje.

\section{La enseñanza y aprendizaje de las matemáticas}

Cuando tenemos en cuenta el tipo de matemáticas que queremos enseñar y la forma de llevar a cabo esta enseñanza debemos reflexionar sobre dos fines importantes de esta enseñanza:

- Que los alumnos lleguen a comprender y a apreciar el papel de las matemáticas en la sociedad, incluyendo sus diferentes campos de aplicación y el modo en que las matemáticas han contribuido a su desarrollo.

- Que los alumnos lleguen a comprender y a valorar el método matemático, esto es, la clase de preguntas que un uso inteligente de las matemáticas permite responder, las formas básicas de razonamiento y del trabajo matemático, así como su potencia y limitaciones.

La perspectiva histórica muestra claramente que las matemáticas son un conjunto de conocimientos en evolución continua y que en dicha evolución desempeña a menudo un papel de primer orden la necesidad de resolver determinados problemas prácticos y su interrelación con otros conocimientos. 
Las matemáticas constituyen el armazón sobre el que se construyen los modelos científicos, toman parte en el proceso de modelización de la realidad, y en muchas ocasiones han servido como medio de validación de estos modelos. Por ejemplo, han sido cálculos matemáticos los que permitieron, mucho antes de que pudiesen ser observados, el descubrimiento de la existencia de los últimos planetas de nuestro sistema solar (Llinares, 1998).

Pregunta $N^{\circ} 1$. ¿Entre qué porcentajes considera usted que utiliza las TIC en sus clases?

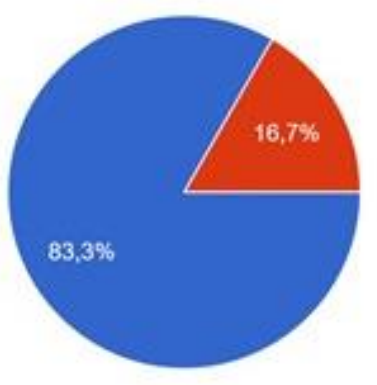

ENTRE $75 \%$ Y $100 \%$

- ENTRE $50 \%$ Y $75 \%$

ENTRE $25 \%$ Y $50 \%$

- $0 \%$

Gráfico 1. Porcentaje de utilización de las TIC en clases

Fuente: Elaboración propia.

Esta realidad indica que la gran mayoría de estudiantes y docentes utilizan las TIC en sus clases, lo cual es coherente a la modalidad de estudios en la que cursan su carrera en el Instituto y permite verificar que si tienen acceso a las TIC mediante Internet para recibir sus clases.

Pregunta $N^{\circ} 2$. Tiene buen dominio para el manejo de las TIC

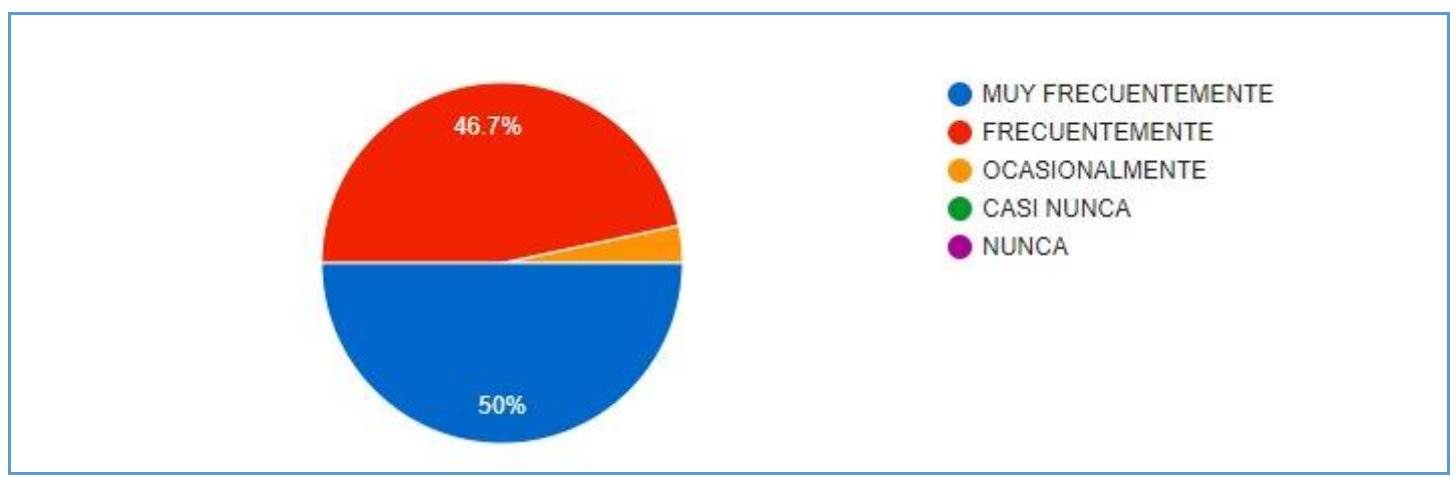

Gráfico 2. Tiene buen dominio para el manejo de las TIC Fuente: Elaboración propia.

Toyo. Integración de las TIC en la enseñanza aprendizaje en línea de la asignatura matemática del ITB 
Dichos resultados indican que la gran mayoría de los estudiantes consideran que tienen unas buenas competencias en el manejo de las TIC. Esta afirmación es importante ya que pueden aceptar rápidamente nuevas formas de aprendizaje que se incluyan en la plataforma de enseñanza en línea.

Pregunta $\mathrm{N}^{\circ}$ 3. En su opinión, la utilización de recursos tecnológicos es importante como apoyo didáctico en los procesos de aprendizaje.

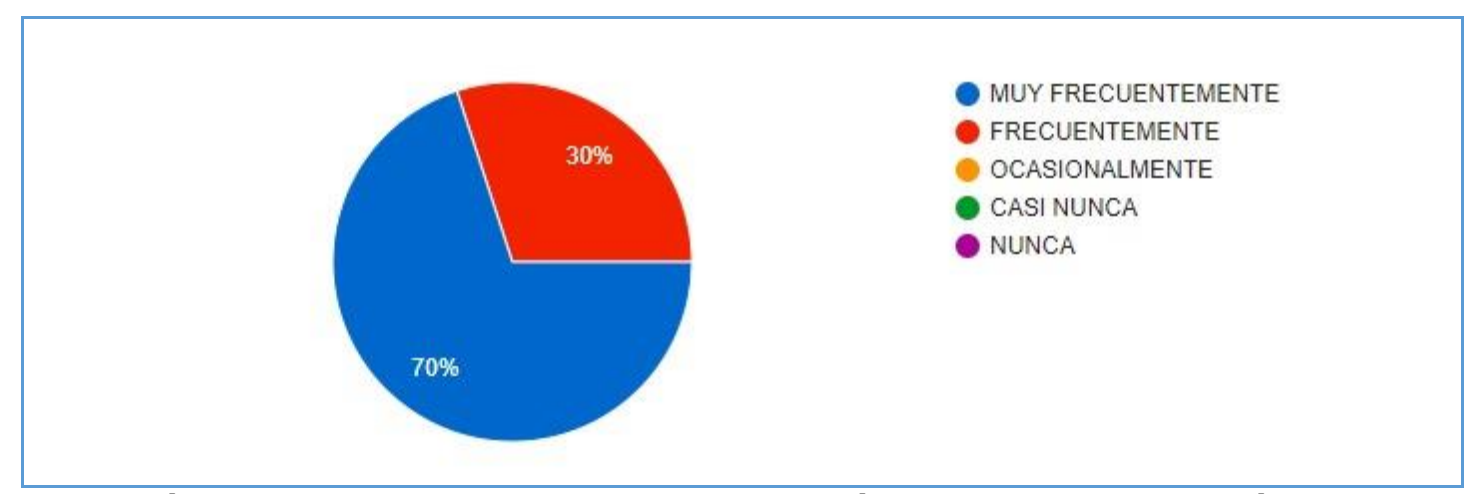

Gráfico 3. Importancia de recursos tecnológicos como apoyo didáctico Fuente: Elaboración propia.

Con este análisis, se puede resumir que todos los encuestados están de acuerdo que los recursos tecnológicos son un soporte importante al momento de adquirir los conocimientos ya que ofrece una diversidad de recursos como complementos para promover el aprendizaje significativo, activo y flexible en el entorno de enseñanza en línea.

Pregunta $\mathrm{N}^{\circ}$ 4. ¿Cree usted que los recursos tecnológicos favorecen la adquisición de aprendizajes, gracias a los ambientes virtuales?

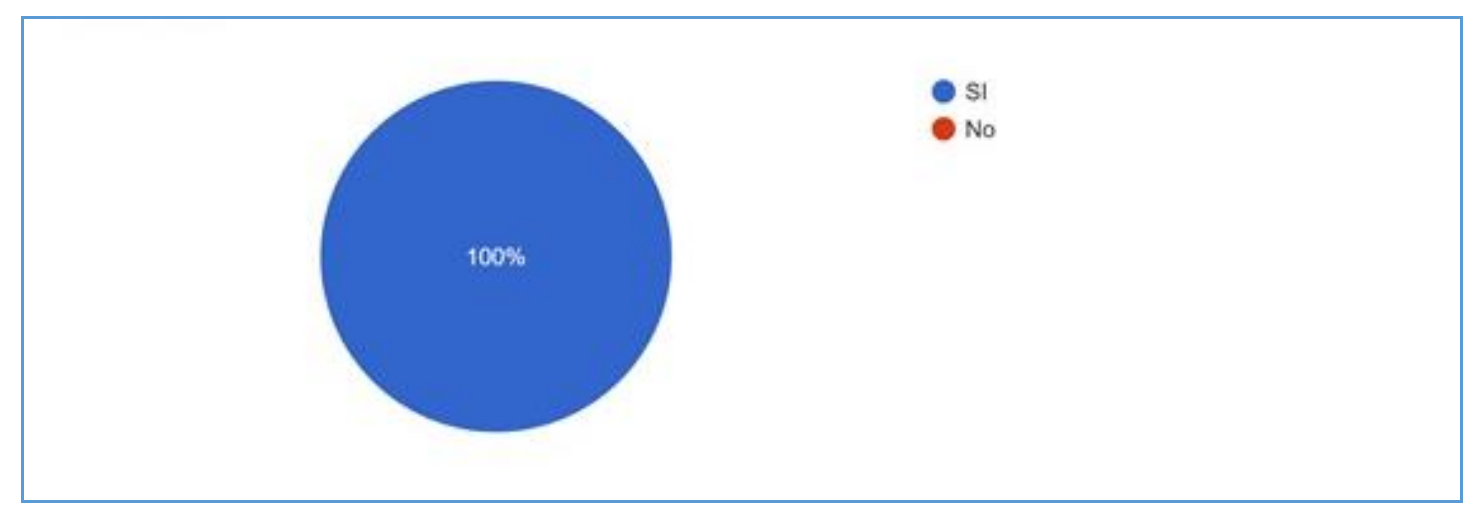

Gráfico 4. Recursos tecnológicos en la adquisición de aprendizajes

Fuente: Elaboración propia.

El $100 \%$ de los encuestados afirman que los recursos tecnológicos favorecen la adquisición de aprendizajes gracias a los ambientes virtuales y que en concordancia con la investigación, benefician a las metodologías de enseñanza en línea. 
Pregunta $\mathrm{N}^{\circ}$ 5. ¿Cuenta la asignatura de matemáticas con recursos digitales (Plan analítico, planificación semanal, videos, cuestionarios) por cada unidad en la plataforma?

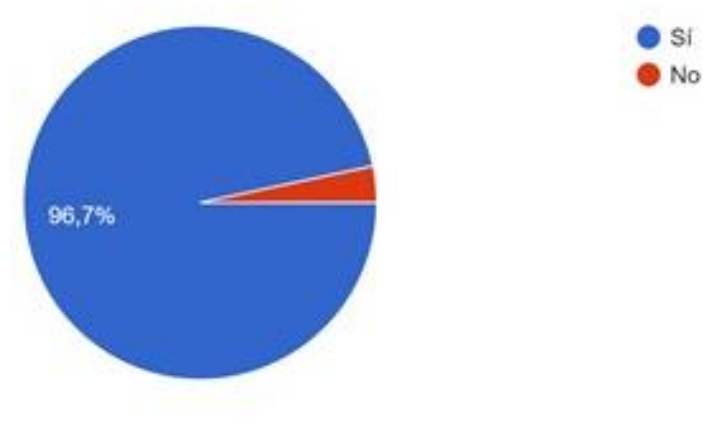

\section{Gráfico 5. Recursos digitales para la asignatura de matemáticas}

Fuente: Elaboración propia.

La gran mayoría considera que la asignatura de matemáticas actualmente cuenta con una serie de medios digitales que los orienta, planifica y prepara en cada uno de las unidades impartidas de la asignatura. Esta postura evidencia los buenos estándares de calidad respecto a la enseñanza en línea que actualmente tiene la institución e incita a mejorar por ese porcentaje restante a través de esta investigación para que en su totalidad se sientan satisfecho.

\section{Conclusiones}

La integración de las TIC de los estudiantes bajo estudio, en el proceso enseñanza aprendizaje en línea de la asignatura matemática, indicaron que la gran mayoría utiliza las TIC en sus clases, lo cual está vinculado a la modalidad de estudios de su carrera en el Instituto y permite verificar que si tienen acceso a las TIC mediante Internet para recibir sus clases. Sin embargo, no hay un uso óptimo de la plataforma y de las herramientas tecnológicas.

El nivel de conocimiento de docentes y estudiantes en la integración de las TIC, inciden notablemente en el proceso de la enseñanza aprendizaje en línea ya que en la actualidad, la tecnología aplicada a la educación brinda herramientas que complementan y que son capaces de ayudar a mejorar el sistema educativo siempre que vengan acompañadas de una buena gestión de las mismas por parte de los docentes, siendo la videoconferencia y la plataforma virtual las de mayor uso, permitiendo al docente orientar a los estudiantes con los contenidos y entorno de la plataforma.

Buena parte de los docentes y estudiantes utilizan herramientas tecnológicas, alegando que usar los objetos virtuales de aprendizajes ayudarán a que las clases de matemáticas sean más prácticas.

Toyo. Integración de las TIC en la enseñanza aprendizaje en línea de la asignatura matemática del ITB 


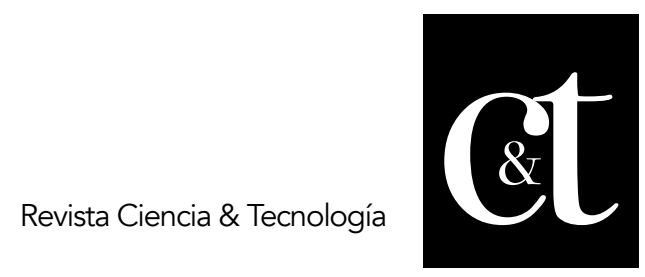

No. 30,30 de abril de 2021

ISSN impreso: 1390 - 6321

ISSN online: 2661 - 6734

Lo previo es muy significativo debido a la naturaleza propia de la materia donde se debe mostrar ejercicios prácticos constantemente, sin dejar de lado ese porcentaje de la población encuestada que requieren de socialización e interacción para el uso adecuado.

\section{Referencias}

Adell, J. (2004), "Internet en el aula: Las WebQuest" Edutec, Revista Electrónica de Tecnología Educativa $17 . \quad$ Recuperado de: http://www.uib.es/depart/gte/edutec-e/revelec17/ADELL 16a.htm

Andersen, J. (1979). Teacher immediacy as a predictor of teaching effectiveness, en D. Nimmo (Ed.), Communication yearbook. New Brunswick, Transaction Books.

Área, M. (2009). Introducción a la Tecnología Educativa. Universidad de La Laguna (España). Licencia Creative Commons.

Bertran, Enric, \& Chamarro, Andrés (2016). Videojugadores del League of Legends: El papel de la pasión en el uso abusivo y en el rendimiento. Adicciones, 28(1),28-34. Recuperado de: https://www.redalyc.org/articulo.oa?id=2891/289144321004

Cabero, J. y Llorente, M.C. (2005). Las plataformas virtuales en el ámbito de la teleformación, en Revista electrónica Alternativas de Educación y Comunicación.

Chaparro, F. (2001). Conocimiento, aprendizaje y capital social como motor de desarrollo. Ciência da Informação, 30(1), 19-31.

CHENOWETH, Iván R., ABRIL-GARCÍA, José Humberto y MEZA-IBARRA, Iván Dostoyewsk. Adoptar nuevas tendencias de elearning XAPI y LRS. Revista de Ciencias de la Educación 2017. 1-2:63-73 recuperado de: Revista_de_Ciencias_de_la_Educación_V1_N2_7

Fernández, C. M. (2018). Guía sobre el uso educativo de los blogs. Universidad Politécnica de Madrid (UPM), GATE, 1-20

García, A. (. (2007). De la Educación a Distancia a la Educación Virtual. Revista de Universidad y sociedad del conocimiento.

García Sánchez, María del Rocío, \& Reyes Añorve, Joaquín, \& Godínez Alarcón, Guadalupe (2017). Las Tic en la educación superior, innovaciones y retos. RICSH Revista Iberoamericana de las Ciencias Sociales y Humanísticas, 6(12), [fecha de Consulta 29 de Julio de 2020]. Recuperado de: https: //www.redalyc.org/articulo.oa?id =5039/503954320013

Hernández, R. Fernández y Baptista, P (2014). Metodología de la Investigación. (Sexta edición ed.). México, DF: Mc Graw Hill.

Lara, L. (2002). Análisis de los recursos interactivos en las aulas virtuales. Argentina: Integración sin barreras en el siglo XXI.

Lázaro y Torres, María Luisa de y Ruiz Palomeque, María Eulalia y González González, María Jesús (2009) La utilización de Hot Potatoes en el Campus Virtual. Moodle como herramienta de autoevaluación. In V Jornada Campus Virtual UCM: Buenas prácticas e indicios de calidad. Universidad Complutense, Madrid, pp. 68-74. ISBN 978-84-7491-968-4.

Llinares, S. y Sánchez, M. V. (1998). Aprender a enseñar matemáticas: Los videos como instrumento metodológico en la formación inicial de profesores. Revista de Enseñanza Universitaria, 13, 29-44. 


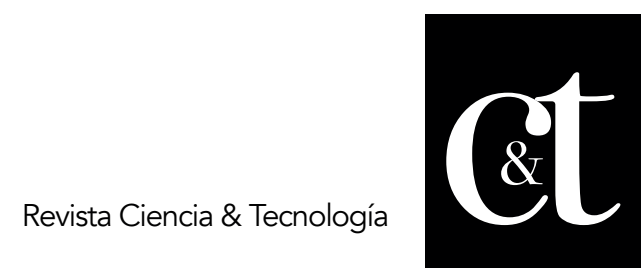

No. 30, 30 de abril de 2021

ISSN impreso: 1390 - 6321

ISSN online: 2661 - 6734

Moya, A (2010). Recursos Didácticos en la Enseñanza. Innovación y Experiencias Educativas. Granada, España.

Rosario, J. (2005). La Tecnología de la Información y la Comunicación (TIC). Su uso como Herramienta para el Fortalecimiento y el Desarrollo de la Educación Virtual. Revista DIM.

Silva, T. y Calichs, E . (2013). El sistema de trabajo metodológico. Disciplinas en la modalidad semipresencial . Cuba: Pedagogía Universitaria.

Toyo. Integración de las TIC en la enseñanza aprendizaje en línea de la asignatura matemática del ITB 